\title{
Protein crystallisation in agarose gel, a cheap and versatile technique
}

\author{
José A. Gavira ${ }^{1}$, Fiora Artusio ${ }^{2}$, Albert Castellví ${ }^{3}$, Roberto Pisano ${ }^{2}$ \\ ${ }^{1}$ LEC, IACT (CSIC-UGR), Avenida de las Palmeras 4, 18100 Armilla, Granada, Spain, ${ }^{2}$ Dpt of Applied Science \& Technology, \\ Politecnico di Torino, 24 corso Duca degli Abruzzi, 10129 Torino, Italy. ${ }^{3}$ Structural Biology, Molecular Biology Institute of \\ Barcelona, Baldiri Reixac 15,08028 Barcelona, Spain
}

jgavira@iact.ugr-csic.es

Crystallization in hydrogels is not a frequent practice in bio-crystallography, although the benefits are multiple: prevents convection and crystal sedimentation, acts as impurity filter, etc., and have been proven to be the cheapest means to produce protein crystals of high quality similar to those obtained under microgravity conditions [1-2]. Moreover, gel grown protein crystals are excellent candidates as seeds to produce crystals of bigger size for neutron diffraction or as media for crystals delivery in serial femtosecond crystallography [3].

Hydrogel should also be considered to exert control over the nucleation and growth processes. In this work we will present our most recent studies on the influence of agarose over the nucleation and growth of protein crystals. Crystal number and size was successfully tuned in a wide range of agarose concentration while keeping constant other conditions. Using five model proteins we demonstrate that the influence of gel content is independent of the protein nature, allowing the mathematical prediction of crystals flux and size with little experimental effort. The convection free environment obtained even at low agarose concentration [4] permits the obtention of high homogeneous micro-crystals slurries (Figure 1) that could be used for serial crystallography application [3] or for the mass production of enzyme crystals for industrial application [5]. Last, we will also show how it allows to explore the phase diagram under a kinetic regime that may facilitate the growth of different polymorphs.

(a)

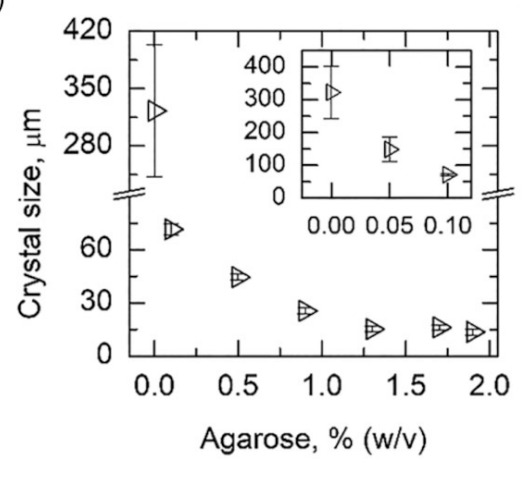

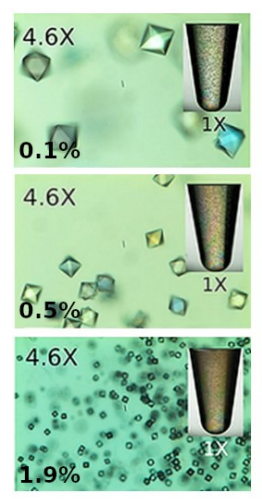

(b)

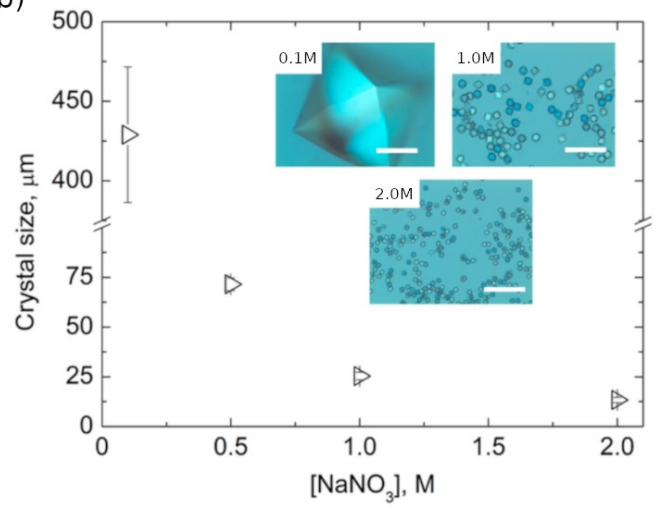

Figure 1. Crystal size and number are fine-tuned using agarose as non-convective media during the crystallization process. (a) Proteinase-K crystals size as a function of agarose concentration and (b) as a function of precipitant concentration at fix agarose concentration of $0.1 \%(w / v)$. Scale bar is $100 \mu \mathrm{m}$.

[1] Gavira, J. A., Otálora, F., González-Ramírez, L. A., Melero, E., Driessche, A. E. S. v., \& García-Ruíz, J. M. (2020). Crystals, 10, 68.

[2] Lorber, B.; Sauter, C.; Théobald-Dietrich, A.; Moreno, A.; Schellenberger, P.; Robert, M.C.; Capelle, B.; Sanglier, S.; Potier, N.; Giegé, R. (2009). Prog. Biophys. Mol. Biol. 101, 13.

[3] Artusio, F.; Castellví, A.; Sacristán, A.; Pisano, R.; Gavira, J.A. (2020). Cryst. Growth Des., 20, 5564.

[4] Garcia-Ruiz, J.M.; Novella, M.; Moreno, R.; Gavira, J.A. (2001). J. Cryst. Growth, 232, 165.

[5] Fernández-Penas, R.; Verdugo-Escamilla, C.; Martínez-Rodríguez, S.; Gavira, J.A. (2021). Cryst. Growth Des., $21,1698$.

\section{Keywords: agarose hydrogel; protein crystal nucleation, serial crystallography}

Supported by project BIO2016-74875-P (MINECO), Spain co-funded by the Fondo Europeo de Desarrollo Regional (FEDER funds), European Union. 